
\title{
BReserch S Suare \\ Three dimensional statistical damage constitutive model of rock based on Griffith strength criterion
}

\section{Song Chen}

Hebei GEO University

Xiuling Cao ( $\square$ caoxiuling@hgu.edu.cn )

Hebei GEO University

\section{Zhao Yang}

Beijing Jiaotong University

\section{Research Article}

Keywords: rock damage, Griffith strength criterion, Weibull distribution, intermediate principal stress, modified damage constitutive model

Posted Date: April 15th, 2021

DOl: https://doi.org/10.21203/rs.3.rs-379746/v1

License: (c) (1) This work is licensed under a Creative Commons Attribution 4.0 International License. Read Full License

Version of Record: A version of this preprint was published at Geotechnical and Geological Engineering on May 22nd, 2021. See the published version at https://doi.org/10.1007/s10706-021-01844-6. 


\title{
Three dimensional statistical damage constitutive model of rock
}

\section{based on Griffith strength criterion}

\author{
Song Chen ${ }^{1}$, Xiuling Cao ${ }^{1, *}$ Zhao Yang ${ }^{2}$ \\ ( ${ }^{1}$ School of Urban Geology and Engineering, Hebei University of Geosciences, Hebei 050031,China; ${ }^{2}$ School of \\ Civil Engineering, Beijing Jiaotong University, Beijing 100044, China)
}

\begin{abstract}
According to the damage mechanics theory and Lemaitre strain equivalence theory, because most rock materials are brittle materials, Griffith strength criterion has good applicability to describe the fracture failure of brittle materials from the perspective of energy. Using a new method to describe the rock micro-element strength based on Griffith strength criterion, and assuming the micro-element strength obeys the Weibull distribution, a true triaxial constitutive model of damage softening reflecting the whole process of rock failure is established. On this basis, the influence of the two parameters in the model on the curve of the constitutive model is analyzed, and the relationship between the two parameters and the intermediate principal stress in the model is established, and the model is revised reasonably. Finally, a true triaxial damage constitutive model of rock is established. The results are in good agreement with the experimental curve, which verifies its validity and rationality. At the same time, the relationship between the damage evolution and the strain and stress is discussed, and the influence of the size of the intermediate principal stress on the relationship is analyzed in detail.
\end{abstract}

Keywords: rock damage; Griffith strength criterion; Weibull distribution; intermediate principal stress; modified damage constitutive model

\section{Introduction}

The mechanism of rock failure is complicated since flaws such as cracks and voids commonly exist in rock. These discontinuous structure surfaces have significant influences on the damage processes and failure characteristics of rock ${ }^{[1-2]}$. Krajcinovic and other scholars ${ }^{[3-6]}$ believe that the internal defects of rock materials are subject to random distribution, and the axial strain strength theory is used to describe the microelement strength of rock. The rock micro-element strength obeys the Weibull distribution, and with the introduction of statistical damage theory, a mesoscopic damage evolution model for the whole process of rock fracture under confining pressure is established. But the axial strain can not fully describe the micro-element strength of rock, and can not directly reflect the bearing capacity of rock, so the model is bound to have some shortcomings. some scholars ${ }^{[7-10]}$ put forward the method of using Drucker-Prager criterion to describe the micro-element strength of rock. Finally, it is found that the Drucker-Prager criterion is more conservative in determining the micro-element strength of rock. Cao and Liao et al. ${ }^{[11-14]}$ noted that the Mohr-Coulomb criterion is based on the tests to set up and can accurately reflect the bearing capacity of rock, and use it to describe the micro-element strength of rock is reasonable and practical, but failed to take into account the influence of intermediate principal stress. Some scholars ${ }^{[15-16]}$ built a damage constitutive model of rock based on Hoek-Brown criterion. It is highly dependent on engineering experience and strong randomness, so there are still some limitations. In addition, some scholars ${ }^{[17-19]}$ have established the statistical damage constitutive model by using the Lade-Duncan strength criterion. Zhu et al. [20] established rock statistical damage softening model based on the SMP (Spatially Mobilized Plane) criterion. The SMP and Lade-Duncan criteria are smooth curves on the $\pi$ plane, and

Corresponding author.

E-mail address: caoxiuling@hgu.edu.cn. 
overcome the defects of nonsmooth curves with singularities. However, the destruction of these criteria is mainly based on the form of pressure, and it is difficult to characterize the tensile failure of brittle rock. At present, the Griffith strength criterion has been widely applied to fracture criterion of brittle materials such as rock $^{[21-22]}$, but it has not been reported in the theoretical damage constitutive model. At the same time, most of the statistical damage models established by predecessors are uniaxial or two-dimensional, and a few are three-dimensional, and the three-dimensional states are pseudo-triaxial $\left(\sigma_{2}=\sigma_{3}\right)$.

In view of this, because most rock materials are brittle materials, Griffith strength criterion has good applicability to describe the fracture failure of brittle materials from the perspective of energy. This paper uses the Griffith strength criterion as a new micro element strength representation method. The effect of intermediate principal stress is taken into account in the three dimensional Griffith strength criterion. Assuming that the strength of the micro-element obeys to the Weibull distribution, a true three-dimensional $\left(\sigma_{2} \neq \sigma_{3}\right)$ state damage constitutive model is established. By using the geometric special point derivation method of constitutive relations with clear physical meaning, the parameters are obtained. The relationship between the parameters and the intermediate principal stress is discussed, and the model is modified reasonably. An example is given to verify its effectiveness and correctness. Finally, the relationship between damage evolution and strain and stress is discussed in detail, and the influence of intermediate principal stress on this relationship is analyzed.

\section{Establishment of statistical constitutive model for rock damage}

\subsection{Rock damage variable and damage Statistic constitutive relation}

According to the strain equivalence hypothesis of Lemaitre ${ }^{[23]}$, the damage constitutive relation of rock is established as follows:

$$
\sigma=\sigma^{*}(1-D)=E \varepsilon(1-D)
$$

Where $E$ : elastic modulus of rock; $\sigma$ : rock nominal stress; $\sigma^{*}$ : rock effective stress; $\varepsilon$ : strain; $D$ : the damage variable of rock.

Because the distribution of microscopic defects in the rock is random, the rock damage variable $D$ can be defined as the ratio of the failure number of rock elements $(n)$ under load and the total number of rock elements $(N)$ without damage, and the range is $0-1$.

$$
D=\frac{n}{N}
$$

According to the theory of probability and statistics, it is assumed that the failure probability distribution law of the micro-element strength of rock is $\mathrm{P}(F)$, therefore, the number of damaged micro-elements obtained by arbitrary small elements $(\mathrm{dF})$ is $N \mathrm{P}(F) \mathrm{dF}$, and when the external load is increased to $F$, the number of damage elements at this point can be expressed as follows:

$$
n=\int_{0}^{F} N P(x) \mathrm{d} x
$$

The substitution of formula (3) into (2):

$$
D=\int_{-\infty}^{F} P(x) \mathrm{d} x
$$

\subsection{Determination of micro-element $\operatorname{strength}(F)$ based on the three-dimensional Griffith strength criterion}

The three-dimensional Griffith strength criterion is that Murrell extended the original two 
dimensional Griffith strength criterion ${ }^{[24]}$. The formula is as follows:

$$
\frac{\left(\sigma_{1}-\sigma_{2}\right)^{2}+\left(\sigma_{2}-\sigma_{3}\right)^{2}+\left(\sigma_{3}-\sigma_{1}\right)^{2}}{24\left(\sigma_{1}+\sigma_{2}+\sigma_{3}\right)}=\sigma_{t}
$$

It can be obtained by the theory of elastic-plastic mechanics:

$$
\begin{gathered}
I_{1}=\sigma_{1}+\sigma_{2}+\sigma_{3} \\
J_{2}=\frac{1}{6}\left[\left(\sigma_{1}-\sigma_{2}\right)^{2}+\left(\sigma_{2}-\sigma_{3}\right)^{2}+\left(\sigma_{3}-\sigma_{1}\right)^{2}\right]
\end{gathered}
$$

The substitution of formula (6), (7) into formula (5):

$$
f\left(I_{1}, J_{2}, \sigma_{\mathrm{t}}\right)=J_{2}-4 I_{1} \sigma_{\mathrm{t}}
$$

Where: $I_{1}$ is the first invariant of stress tensor, $J_{2}$ is the stress deviator second invariant, and $\sigma_{\mathrm{t}}$ is rock tensile strength.

The three-dimensional Griffith strength criterion takes into account the influence of confining pressure and reflects the tensile failure of rock under external compressive stress. The strength of the element is described by $F$ :

$$
F=\frac{J_{2}}{4 I_{1}}
$$

Under different confining pressures, according to the generalized Hooke's law and formula (1) can be obtained:

$$
\begin{aligned}
& \sigma_{1}^{*}=E \varepsilon_{1}+v\left(\sigma_{2}^{*}+\sigma_{3}^{*}\right) \\
& \sigma_{i}^{*}=\sigma_{i} /(1-D) i=1,2,3
\end{aligned}
$$

Where: $\sigma_{1}, \sigma_{2}, \sigma_{3}$ is nominal stress, $\sigma_{1}^{*}, \sigma_{2}^{*}, \sigma_{3}^{*}$ represents effective stress, $\varepsilon_{1}$ is axial strain, $v$ is Poisson's ratio.

The substitution of formula (10), (11) into formula (9) :

$$
F=\frac{E \varepsilon_{1}\left(\left(\sigma_{1}-\sigma_{2}\right)^{2}+\left(\sigma_{2}-\sigma_{3}\right)^{2}+\left(\sigma_{1}-\sigma_{3}\right)^{2}\right)}{24\left(\sigma_{1}-v\left(\sigma_{2}+\sigma_{3}\right)\right)\left(\sigma_{1}+\sigma_{2}+\sigma_{3}\right)}
$$

\subsection{Establishment of rock damage evolution model}

By using the Weibull probability distribution of two parameters, assuming that the micro-element strength of rock obeys the Weibull distribution law, the distribution law of the density function is ${ }^{[19]}$ :

$$
P(F)=\frac{m}{F_{0}}\left(\frac{F}{F_{0}}\right)^{m-1} \exp \left[-\left(\frac{F}{F_{0}}\right)^{m}\right]
$$

Where: $F$ : rock micro-element strength; $m$ and $F_{0}$ are two distribution parameters, and they can reflect their mechanical properties.

The formula (13) is substituted (4):

$$
D=\int_{-\infty}^{F} P(x) \mathrm{d} x=1-\exp \left[-\left(\frac{F}{F_{0}}\right)^{m}\right]
$$

The formula (14) is substituted (1):

$$
\sigma_{1}=E \varepsilon_{1} \exp \left[-\left(\frac{F}{F_{0}}\right)^{m}\right]+v\left(\sigma_{2}+\sigma_{3}\right)
$$

\subsection{Determination of model parameters}

At present, the methods of determining statistical parameters in literature are commonly used linear fitting method ${ }^{[10-11,15,17,20]}$, such as peak point method ${ }^{[7-8]}$ and inversion analysis method 
${ }^{[8,25]}$ is not used very much. The linear fitting method is based on the discrete points of the existing test data to obtain the required parameter values, and the processing method is simple and easy. But the peak point method needs to differentiate the implicit constitutive equation, and the processing is more complex, and the relation between the stress-strain curve and the geometric point is relatively clear, and the inverse analysis method requires a great amount of experimental data. In the past, the constitutive equation was linearized, and the parameters of the model were determined by the fitting of the experimental curve, this method is not clear about the physical meaning of the model parameters, and occasionally causes negative damage values before the peak and the post peak data point is too small to fail. Therefore, this paper adopts the geometric key point derivation method with general and definite physical meaning. First of all, the geometric constraints on the relation of the constitutive equation before the peak load (including the peak load point) are as follows:

(1) $\varepsilon_{1}=0, \sigma_{1}=0$; (2) $\varepsilon_{1}=0, \frac{\mathrm{d} \sigma_{1}}{\mathrm{~d} \varepsilon_{1}}=E$; (3) $\varepsilon_{1}=\varepsilon_{\text {peak point }}, \sigma_{1}=\sigma_{\max } ;(4) \varepsilon_{1}=\varepsilon_{\text {peak point }}$, $\frac{\mathrm{d} \sigma_{1}}{\mathrm{~d} \varepsilon_{1}}=0$,

According to the above conditions, the parameter formula of true triaxial state can be given :

$$
\left\{\begin{array}{l}
m=\frac{1}{\operatorname{In} \frac{E \varepsilon_{\text {peakpoint }}}{\sigma_{\text {max }}-v\left(\sigma_{2}+\sigma_{3}\right)}} \\
F_{0}=\left(\frac{F^{m}}{\operatorname{In} \frac{E \varepsilon_{\text {peakpoint }}}{\sigma_{\text {max }}-v\left(\sigma_{2}+\sigma_{3}\right)}}\right)^{1 / m}=F \times m^{1 / m}
\end{array}\right.
$$

\section{Modification and example verification of statistical constitutive model for rock damage}

\subsection{The influence of two parameters $\left(m, F_{0}\right)$ on the constitutive model in Weibull distribution}

From the above rock damage statistical constitutive model, it can be seen that the two parameters $\left(m\right.$ and $\left.F_{0}\right)$ have some influence on rock mechanics and deformability. There are four parameters $\left(E, v, m\right.$ and $\left.F_{0}\right)$ in the model proposed in this paper, according to the data of literature [26], modulus of elasticity $E=90 \mathrm{GPa}$, poisson ratio $v=0.15$, the parameter $m$ and $F_{0}$ can be obtained by the formula (20). As shown in Figure 1 Figure 2.

(1) When the parameter $m$ is constant in Figure1, the damage constitutive curve of rock varies with $F_{0}$. As can be seen from the Figure1, with the increase of $F_{0}$, the peak strength and the corresponding strain of the constitutive curves increase. The linear elastic part before the peak is almost not affected by $F_{0}$, and the non-linear elastic part before the peak increases with the $F_{0}$, and the curvature is constantly slowing down. Post the peak curve increases with $F_{0}$, the decline rate of curve decreases continuously and even shows an upward trend, which indicates that $F_{0}$ reflects the macro statistical average intensity of rock.

(2) When the parameter $F_{0}$ is constant in Figure2, the damage constitutive curve of rock varies with the parameter $m$. As can be seen from the Figure2, the peak intensity and peak strain 
increase with the $m$ increases before the strain equal to 0.0047 . The change of pre-peak curvature is relatively smooth, and the post-peak curvature changes rapidly. This shows that parameter $m$ has a great influence on the transformation between brittle and ductile rocks, and it has little effect on the elastic part before the curve peak, which indicates that $m$ reflects the concentration of the micro-element intensity distribution in the rock.

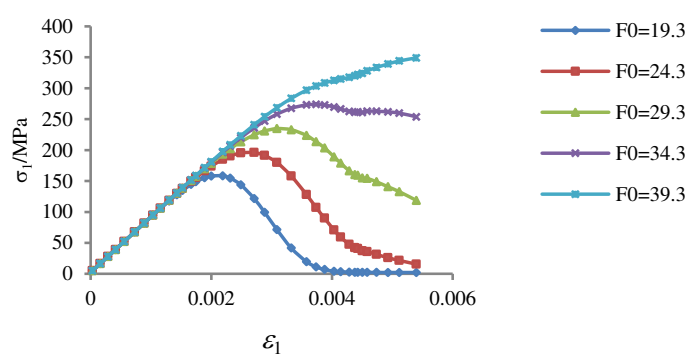

Fig.1 The effect on constitutive model of the parameter $F_{0}$

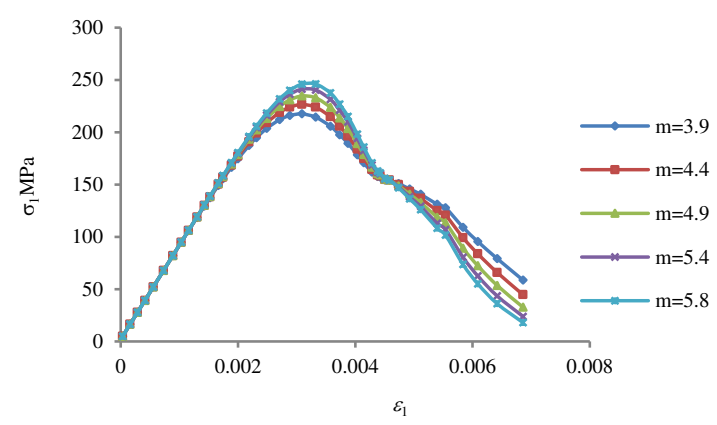

Fig. 2 The effect on constitutive model of the parameter $m$

A large number of rock three axis tests show that the peak strength of rock constitutive curve increases with the increase of confining pressure, especially, it has a great influence on the hardening and softening stage of rock, and the change of confining pressure can transform rock from brittle to ductile. Can be seen from the above analysis, the confining pressure not only on the stress-strain curve but the two parameters have influence on constitutive curve. Therefore, laid the foundation for further amendments to the proposed statistical damage constitutive model.

\subsection{Example verification}

To verify the validity and accuracy of the model based on the Griffith strength criterion, according to the data of literature [27], and the rock mechanics parameters are: modulus of elasticity $E=733 \mathrm{GPa}$, poisson ratio $v=0.204$, the parameter $m$ and $F_{0}$ can be obtained by the formula (20). The damage constitutive model is established in two steps. Firstly, the statistical damage constitutive relationship of rock under three-axial different confining pressure is established. Second, due to the influence of confining pressure on damage and failure process of rock, the relationship between confining pressure and constitutive model parameters is established, and the model is modified. Finally, the modified constitutive model of rock damage softening is obtained. 
Using the true triaxial test data in reference [27], according to the influence of confining pressure on the parameters $\left(m\right.$ and $\left.F_{0}\right)$, the relationship between the two parameters and the intermediate principal stress is established. Taking the intermediate principal stress $\sigma_{2}$ as the horizontal axis and the two parameters as the longitudinal axis, the functional relationship is established. The specific model parameters and fitting relationships are shown in Table 1, Figure 3. The modified damage constitutive equation can be obtained by substituting the fitting function in Fig.3. Modified parameters of constitutive model under different confining pressures is shown in Table 2. The following results can be obtained by replacing all the known parameters and the calculated parameters into the formula (15).

Table 1 Parameters of constitutive model under different confining pressures

\begin{tabular}{ccc}
\hline$\sigma_{2} / \mathrm{MPa}$ & $m$ & $F_{0} / \mathrm{MPa}$ \\
\hline 1 & 1.047 & 1.421 \\
2 & 1.559 & 1.379 \\
3 & 1.758 & 1.656 \\
4 & 2.695 & 1.503 \\
5 & 3.589 & 1.559 \\
\hline
\end{tabular}

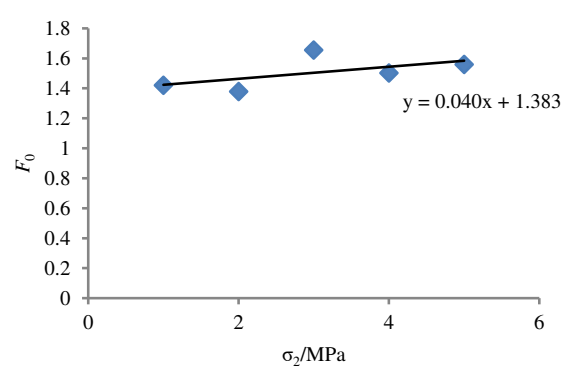

(a)

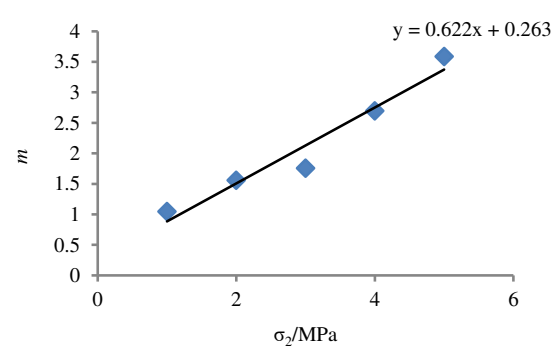

(b)

Fig.3 Fitting relationship between the two parameters and confining pressure

Table 2 Modified parameters of constitutive model under different confining pressures

$\sigma_{2} / \mathrm{MPa} \quad m \quad F_{0} / \mathrm{MPa}$




\begin{tabular}{llc}
\hline 1 & 0.885 & 1.423 \\
2 & 1.507 & 1.463 \\
3 & 2.129 & 1.503 \\
4 & 2.751 & 1.543 \\
5 & 3.373 & 1.583 \\
\hline
\end{tabular}

It can be seen from Figure 4 that the constitutive model considering the effect of intermediate principal stress based on Griffith strength criterion is in good agreement with the experimental curve, which shows that the proposed model is effective and reasonable. The model also shows the following characteristics: 1) This model fully reflects the influence of different confining pressure states on the softening characteristics and strength of rock, especially the effect of intermediate principal stress on the mechanical properties of rock. With the increases of intermediate principal stress, the strength of rock increases, which is in agreement with the experimental results and the conclusions of Takahashi et al ${ }^{[28]}$. 2) The process of rock damage and failure is affected by the micro-element strength of rock and the stress state of rock. Many previous pseudo-triaxial $\left(\sigma_{2}=\sigma_{3}\right)$ damage models believe that rock failure is caused by the stress difference of rock $\left(\sigma_{1}-\sigma_{3}\right)$. However, the intermediate principal stress is also a factor that can not be ignored in the research model. 3) In the constitutive model, the two parameters $\left(m, F_{0}\right)$ not only influence the shape of the constitutive model, but also are sensitive to the confining pressure. Therefore, the relationship between the two parameters and the intermediate principal stress is established, and the constitutive model is corrected. Through the above three points, it is proved that the damage constitutive model is effective.

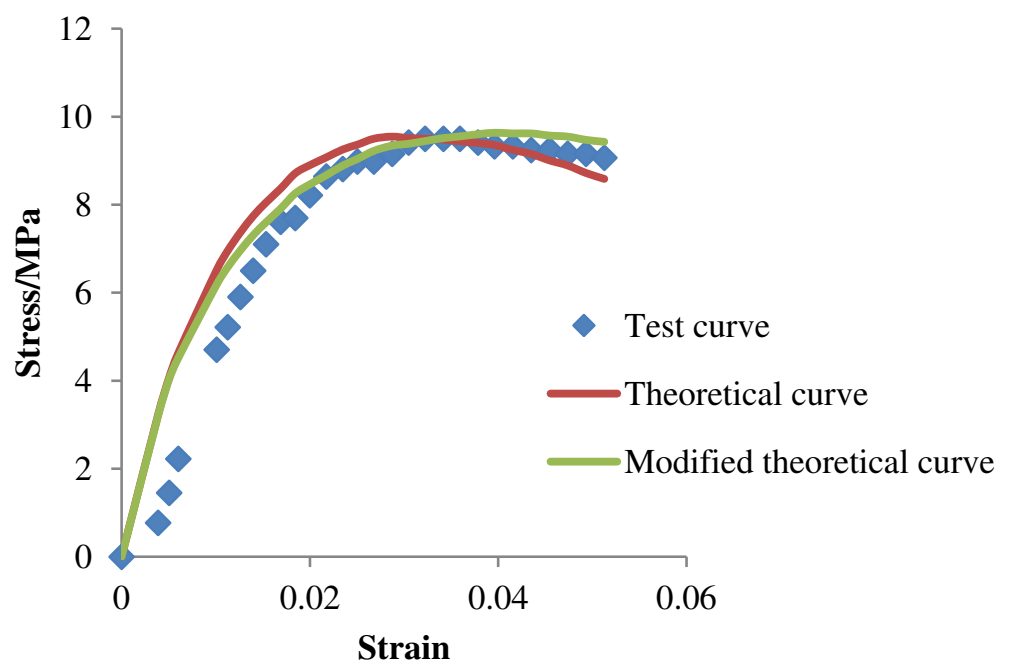


(a) $\sigma_{2}=1 \mathrm{MPa}, \frac{\sigma_{2}}{\sigma_{3}}=1$

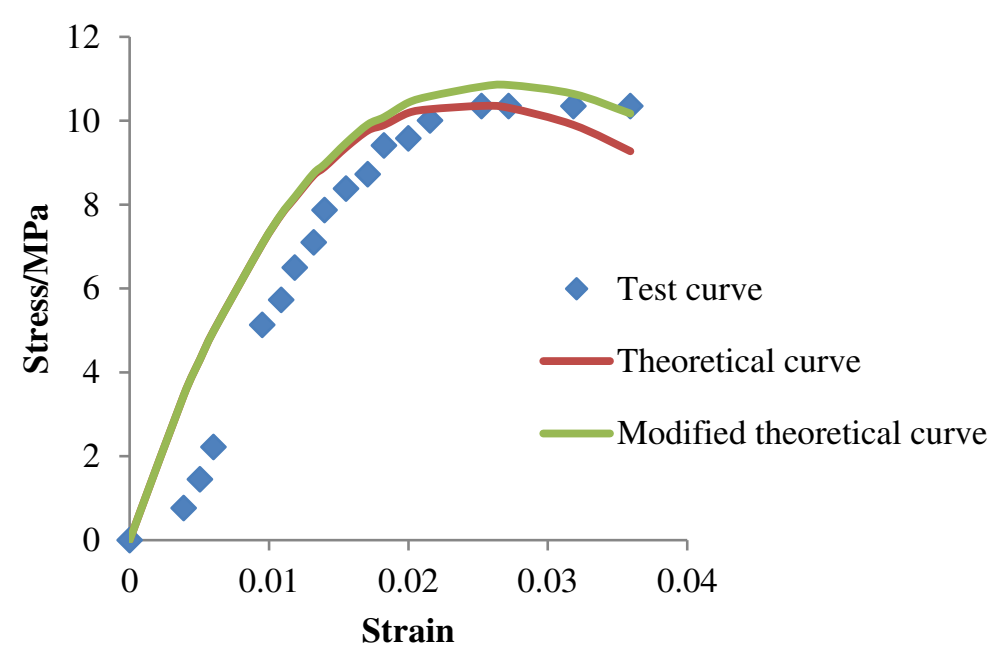

(b) $\sigma_{2}=2 \mathrm{MPa}, \frac{\sigma_{2}}{\sigma_{3}}=2$

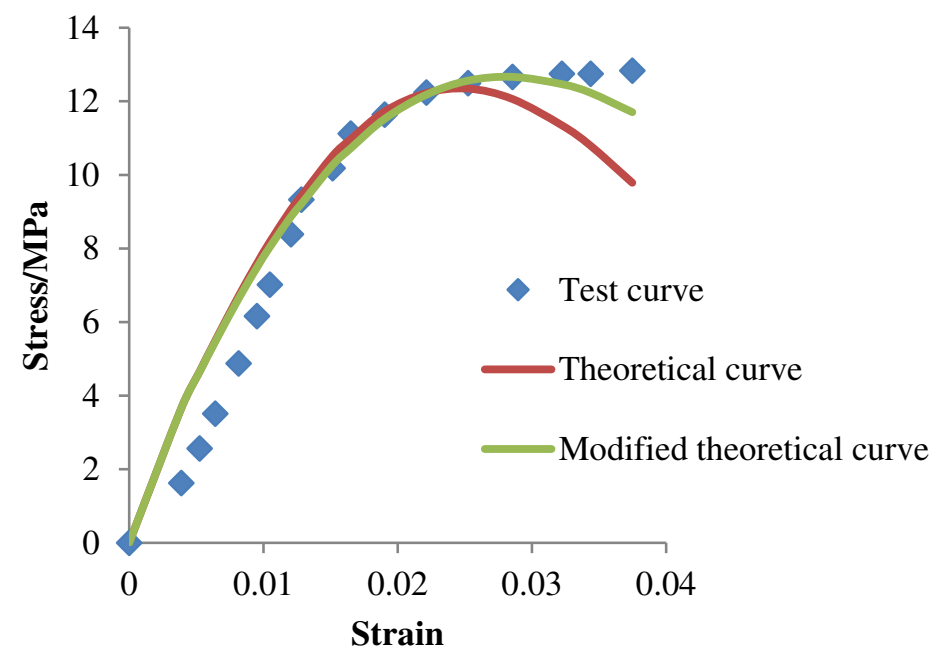

(c) $\sigma_{2}=3 \mathrm{MPa}, \frac{\sigma_{2}}{\sigma_{3}}=3$ 


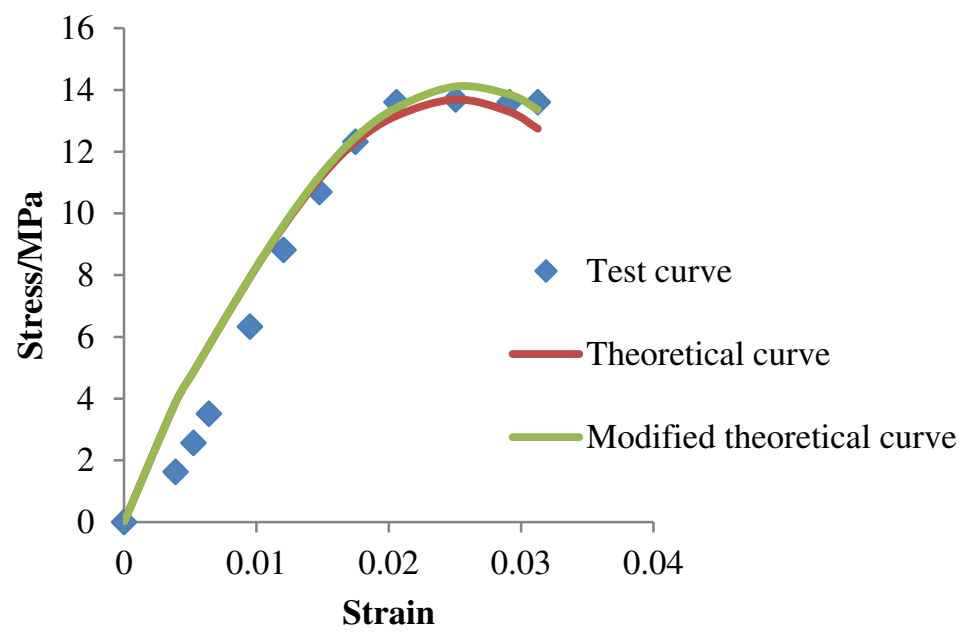

(d) $\sigma_{2}=4 \mathrm{MPa}, \frac{\sigma_{2}}{\sigma_{3}}=4$

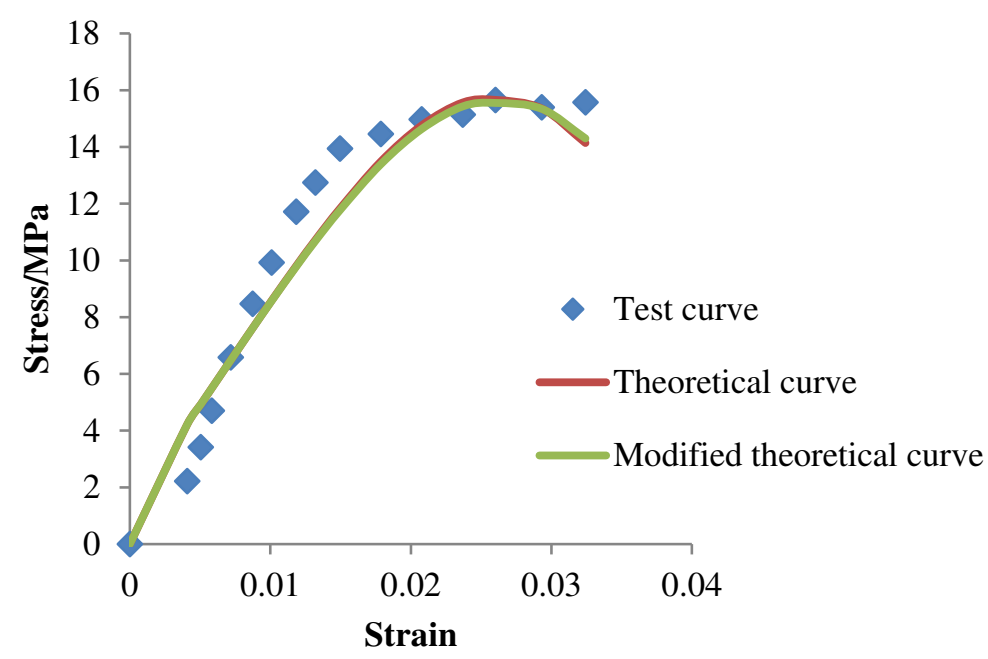

(e) $\sigma_{2}=5 \mathrm{MPa}, \frac{\sigma_{2}}{\sigma_{3}}=5$

Fig.4 Comparison of experimental curve and theoretical curve

Figure 5 shows the theoretical curve of $\varepsilon_{1}-D$, it can be seen from the figure, the shape of the curve is roughly "s" type. With the increases of the intermediate principal stress, the slope of the curve shows a tendency of slow down. The strain is in the range of $0-0.005$, and the damage under different intermediate principal stresses is almost zero. It is considered that under low stress conditions, due to the existence of a large number of natural micro-cracks in the rock, these natural micro-cracks are in the stage of compaction and closure, and no obvious new damage cracks have appeared. Therefore, the damage is almost zero in this range. With the increases of the force and the elongation of the strain, the slope of the damage curve is different under different intermediate principal stresses, and the damage weaken with the increases of the intermediate 
principal stress. It is considered that the cause of this phenomenon may be that the magnitude of the intermediate principal stress restricts the crack initiation, expansion and transfixion, and the greater the intermediate principal stress is, the stronger the binding action is. When the intermediate principal stress $\sigma_{2}=1 \mathrm{MPa}$, the damage evolution curve is upper convex type after strain is about 0.005 . The damage development rate is gradually decreasing, and the curve tends to a fixed value, which shows the characteristics of ductile failure of rock. With the increases of the intermediate principal stress, the damage evolution curve changes from the upper convex type to the downward concave type. The proportion of parts after the inflection point of the upper part of the curve gradually decreases, which shows that the ductility of rock weaken. When the same damage occurs, the strain elongation increases with the increases of confining pressure, which accords with the law of the influence of confining pressure on deformation and failure, and this relationship fully indicates that the damage of rock is affected by stress state.

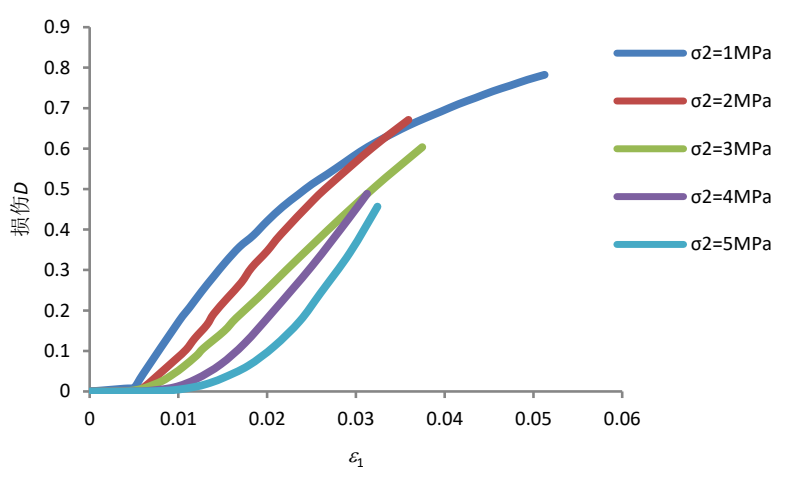

Figure 5 Theoretical curves of $\varepsilon 1-D\left(\sigma_{3}=1 \mathrm{MPa}\right)$

Figure 6 shows the theoretical curve of $\sigma_{1}-D$, and the shape of the curve is roughly a fall down "U" shape. The damage of curves under different intermediate principal stresses is almost zero in a certain range of stresses. It is considered that the natural original cracks in rock are closing gradually under low stress condition, but no new microcracks have been produced. With the increases of intermediate principal stress, the damage rate slows down. It is considered that this may be due to the size of the intermediate principal stress that constrains the development of the crack development in the rock. When the intermediate principal stress is different, the stress required for the corresponding damage at the curve turning point is also different. With the increases of the intermediate principal stress, the damage curve continuously expand outwards. When the initial damage occurs the stress required is also different, which shows that the larger 
the intermediate principal stress is, the greater the initial damage stress required is.

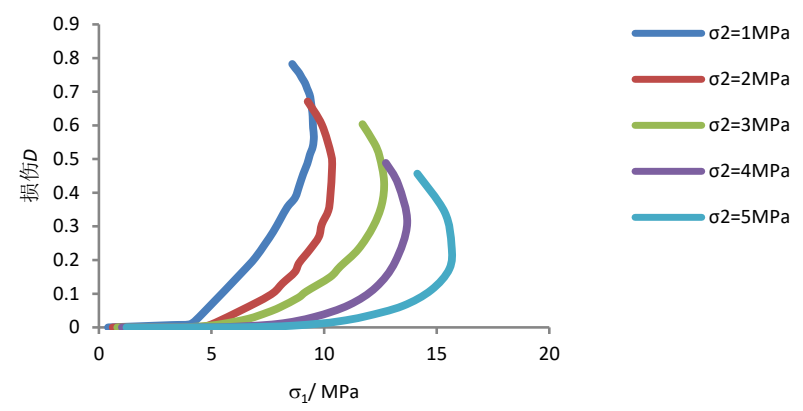

Figure 6 Theoretical curves of $\sigma_{1}-D\left(\sigma_{3}=1 \mathrm{MPa}\right)$

\section{Conclusion}

(1) This model takes into account the effect of intermediate principal stress on rock characteristics, and the damage constitutive model of rock under true triaxial state can well simulate the stress-strain relationship of rock. The effectiveness of using Griffith strength criterion to describe the mechanical properties of rock materials is verified.

(2) The true triaxial damage constitutive model curve based on Griffith strength criterion is in good agreement with the experimental curve, which can reflect the softening and failure process of rock under complex stress state, and verify the validity and correctness of the model. In order to optimize the model, the relationship between the two parameters $\left(m, F_{0}\right)$ and intermediate principal stress is discussed, and the modified constitutive model is closer to reality. The model also fully reflects the damage of rock during softening and failure is not only related to the selection of micro-element strength of rock, but also to the stress state of rock.

(3) The relationship between damage evolution and strain and stress of rock is discussed. Under different confining pressures, the rate of damage evolution and the initial stress required when the damage begins to occur are all affected by the intermediate principal stress.

Acknowledgments This research is sponsored by the Fundamental Research Funds for the Central Universities of China No. 2018YJS127 and the National Natural Science Foundation of China under Contract No. 51278046.

\section{References}

[1] Liu Hongyan, Li Junfeng (2016) A method for calculating damage variable of rock mass with non-persistent joints. Rock and Soil Mechanics, 37(Supp.1): 95-100.

[2] Liu Hongyan, Yang Yan, Li Junfeng, et al (2016) Dynamic damage constitutive model for rock mass with non-persistent joints based on the TCK model. Explosion and Shock Waves, 36(3): 319-325. 
[3] Krajcinovic D, Silva M A G (1982) Statistical aspects of the continuous damage theory. Int J Solids Structures, 18(7):551-562.

[4] Tang Chunan (1993) Catastrophe in rock failure process. Beijing: China Coal Industry Publishing House.

[5] Yang Shengqi, Xu Weiya, Wei Lide, et al (2004) Rock damage constitutive model and experimental study under uniaxial compression. Hohai University: Natural Science, 32(2): 200-203.

[6] Zeng Sheng, Yang Shijiao, Zhang Xinhua, et al (2005) Statistical constitutive model for limestone rock damage under uniaxial compression and its experimental study. Journal of NanhuaUniversity(Science and Technology), 19(1): 69-95.

[7] Wang Z L, Li Y C, Wang J G (2007) A damage-softening statistical constitutive model considering rock residual strength. Computers \& Geosciences, 33(1):1-9.

[8] Deng J, Gu D (2011) On a statistical damage constitutive model for rock materials. Computers \& Geosciences, 37(2):122-128.

[9] Zhao H, Zhang C, Cao W G, et al (2016) Statistical meso-damage model for quasi-brittle rocks to account for damage tolerance principle. Environmental Earth Sciences, 75(10): 862.

[10] Cao Wengui, Fang Zulie, Tang Xuejun (1998) A study of statistical constitutive model for soft and damage rock. Chinese Journal of Rock Mechanics and Engineering, 17(6): 628-633.

[11] Cao Wengui, Zhang Sheng (2005) Study on the statistical analysis of rock damage based on Mohr-Coulomb criterion. Journal of Hunan University(Natural Sciences), 32(1): $43-47$.

[12] Liao Hualin, Li Gensheng (2005) Statistical constitutive model fou rock damage based on Mohr-Coulomb criterion. Oil Drilling Technology, 27 (6): 85-87.

[13] Li X, Cao W G, Su Y H (2012) A statistical damage constitutive model for softening behavior of rocks. Engineering Geology, 143-144:1-17.

[14] Cao Wengui, Li Xiang, Zhao Heng (2007) Damage constitutive model for strain-softening rock based on normal distribution and its parameter determination. Journal of Central South University of Technology, 14(5):719-724.

[15] Shi Chong, Jiang Xinxing, Zhu Zhende et al (2011) Study of rock damage constitutive model and discussion of its parameters based on Hoek-Brown criterion. Chinese Journal of Rock Mechanics and Engineering, 30(1): 2647-2652.

[16] Ma L J, Liu X Y, Fang Q, et al (2013) A new Elasto-Viscoplastic damage model combined with the generalized Hoek-Brown failure criterion for bedded rock salt and its application. Rock Mech Rock Eng, 46: 53-66.

[17] Tian Zhenyuan, Wang Wei, Zhu Qizhi, et al (2014) A statistical damage constitutive model and its modifying method based on Lade-Duncan failure criterion. Science Technology and Engineering, 14(35): 104-108.

[18] Tian Z Y, Wang W, Li X H, et al (2014) A statistical damage constitutive model for brittle rocks based on the Lade-Duncan failure criterion. Advanced Materials Research, 2014, 919-921:632-636.

[19] Ewy R (1999) Wellbore-stability prediction by use of a modified Lade criterion. SPE Drill Completion, 14(2): 85-91.

[20] Zhu Jianming, Cheng Haifeng, Yao Yangping (2013) Statistical damage softening model of fractured rock based on SMP failure criterion and its application. Chinese Journal of Rock Mechanics and Engineering, 32(Supp. 2): 3160-3168. 
[21] Liu Quansheng, Wei Lai, Liu Xuewei, et al (2017) A revised empirical method for predicting crack initiation based on Griffith strength criterion. Chinese Journal of Rock Mechanics and Engineering, 36(7):1561-1569.

[22] Huang K, Shimada T, Ozaki N, et al (2017) A unified and universal Griffith-based criterion for brittle fracture. International Journal of Solids \& Structures, 128: 67-72.

[23] Lemaitre J (1984) How to use damage mechanics. Nuclear Engineering and Design, 80(3): 233-245.

[24] Zhang Qing, Du Jing, Yu Xuefu (1997) Fundamentals of rock mechanics. Beijing: China Railway Publishing House, 62-70.

[25] Liu Chengxue, Yang Linde, Cao Wengui (2007) A statistical damage softening constitutive model for rock and back analysis of its parameters. Chinese Journal of Underground Space and Engineering, 3(3): 453-457.

[26] Zhou, W. Y. et al (1989) Advances in rock mechanics. Shenyang: Northeast Institute of Technology Press.

[27] Liu Handong, Cao Jie (2008) Test and study on influence of intermediate main stress to characteristic of rock mechanics. Yellow River, 30(1): 59-60.

[28] Takahashi, M., \& Koide, H (1989) Effect of the intermediate principal stress on strength and deformation behavior of sedimentary rocks at the depth shallower than $200 \mathrm{~m}$. International Symposium on Rock at Great Depth. 
Figures

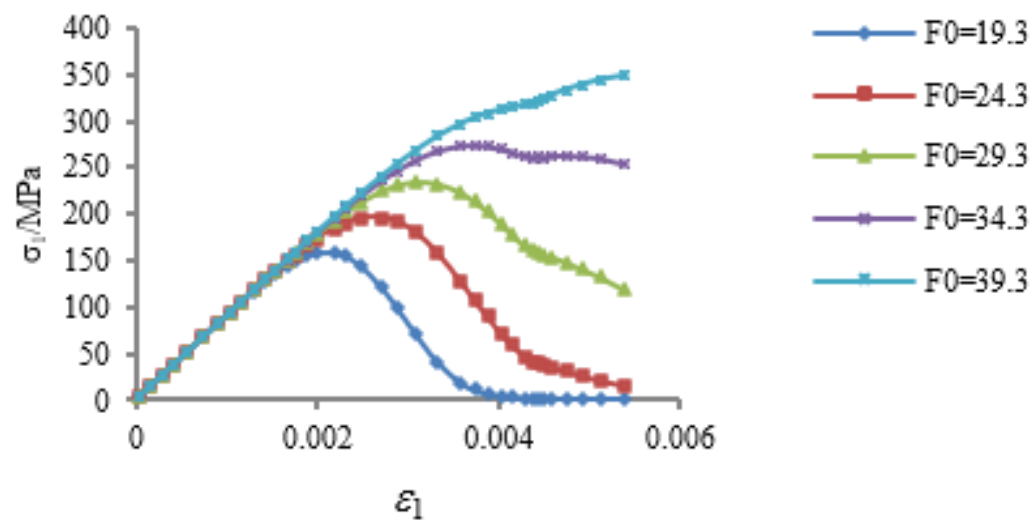

Figure 1

The effect on constitutive model of the parameter F0

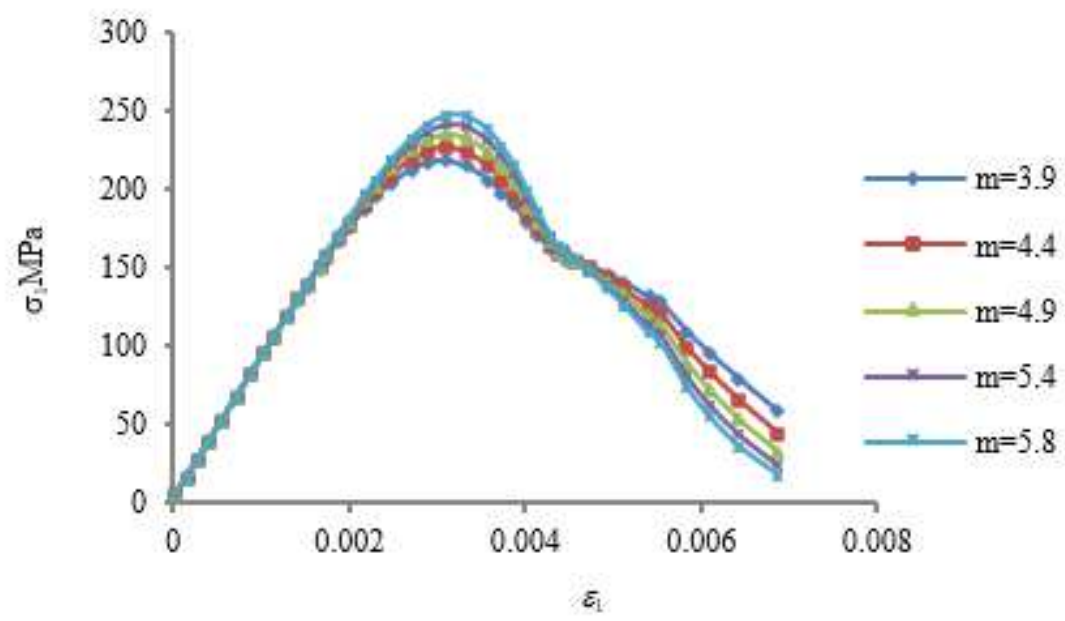

Figure 2

The effect on constitutive model of the parameter $\mathrm{m}$ 


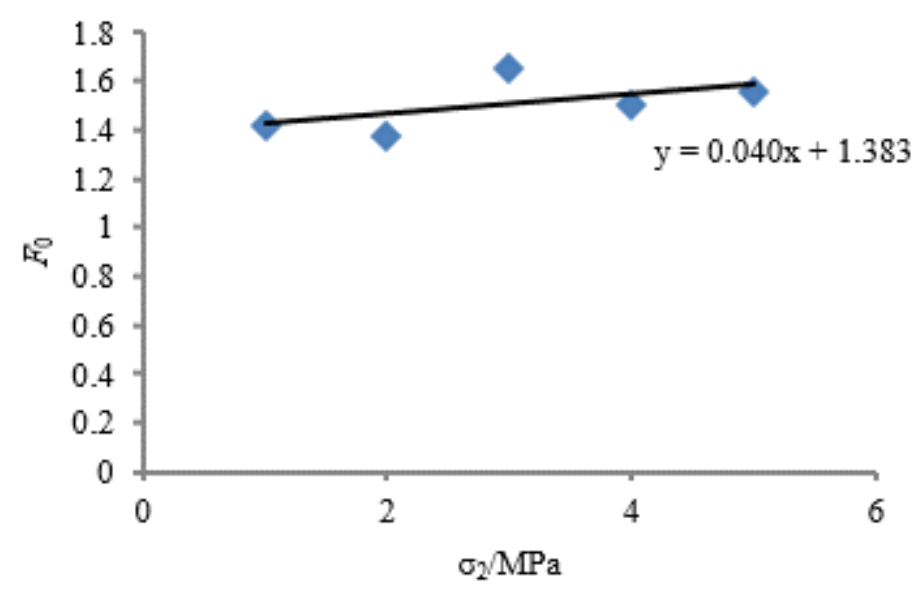

(a)

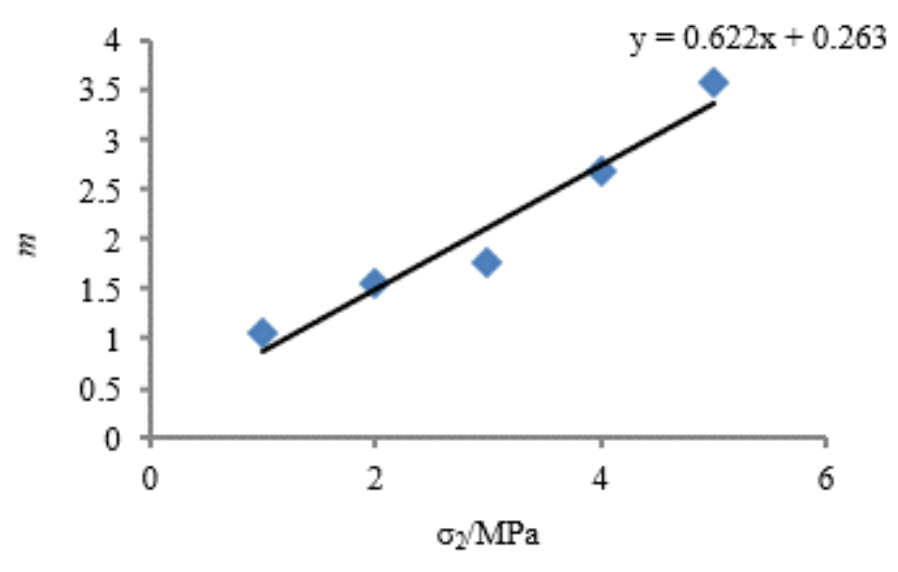

(b)

Figure 3

Fitting relationship between the two parameters and confining pressure 

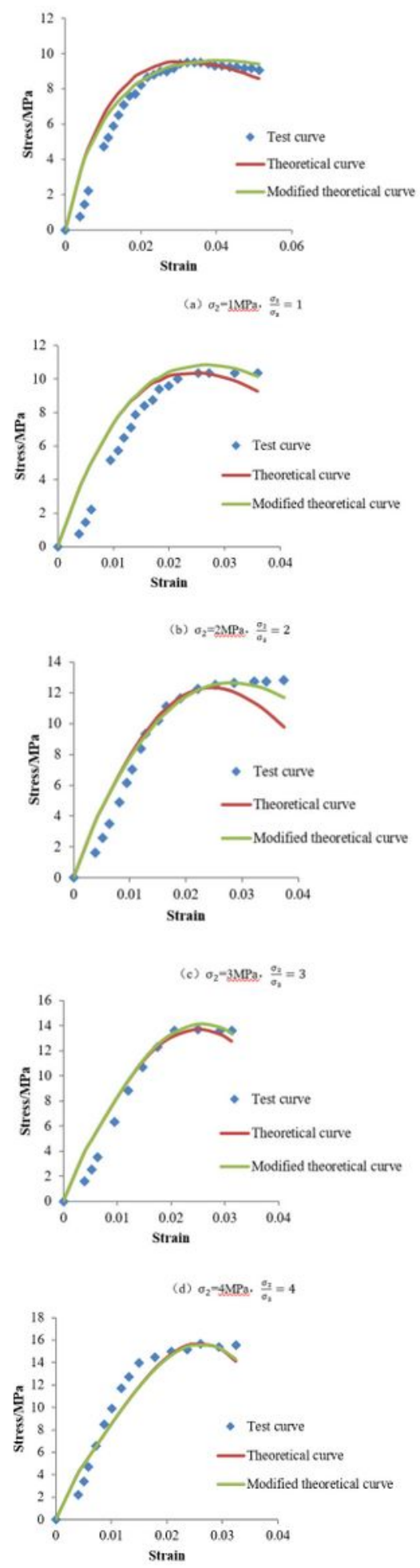

(e) $\sigma_{2}=5 \mathrm{MPP} \cdot \frac{\sigma_{2}}{\alpha_{0}}=5$

\section{Figure 4}

Comparison of experimental curve and theoretical curve 


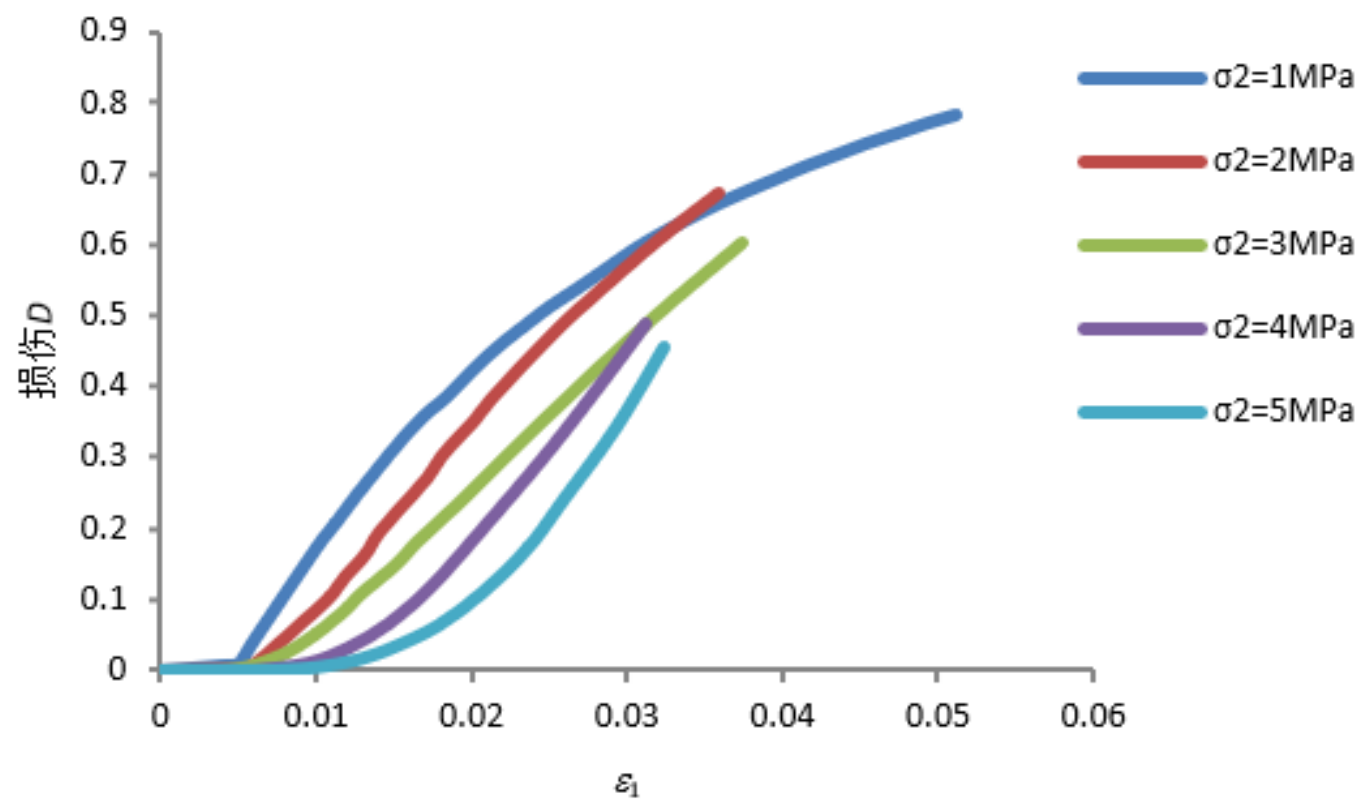

Figure 5

Theoretical curves of $\varepsilon 1-D(\sigma 3=1 \mathrm{MPa})$
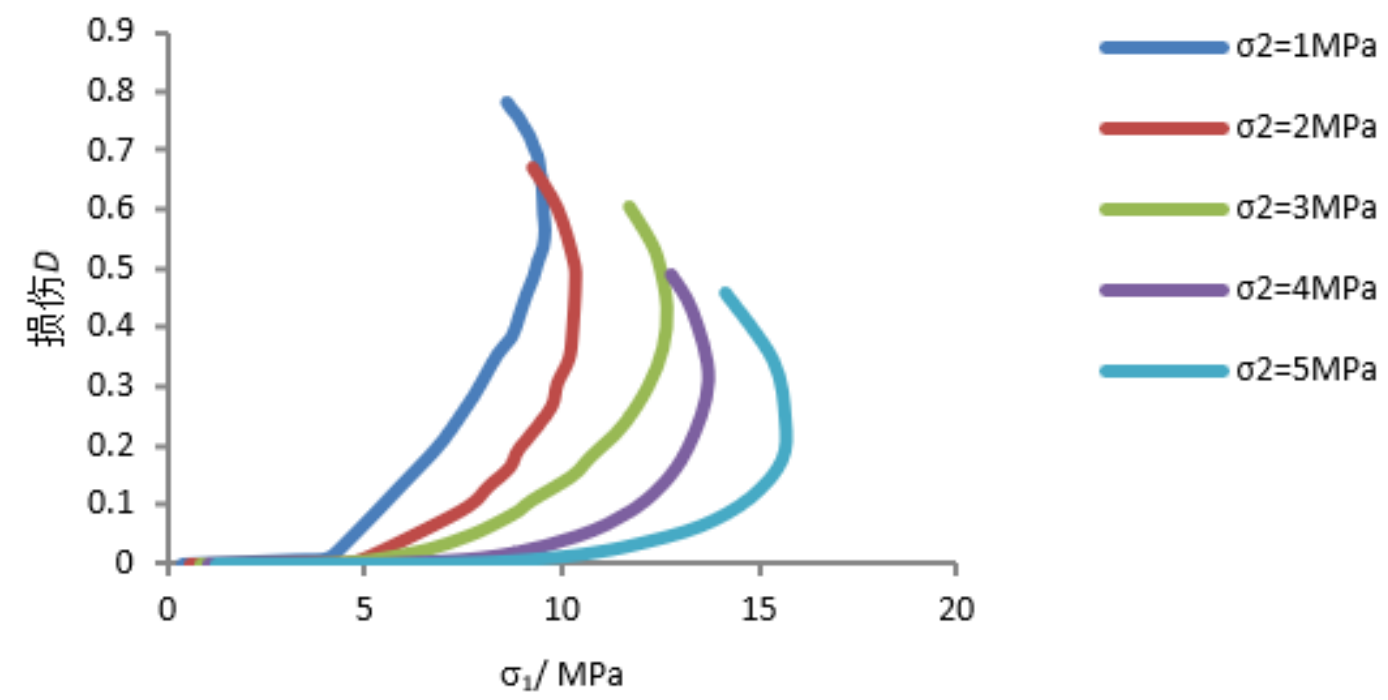

Figure 6

Theoretical curves of $\sigma 1-D(\sigma 3=1 \mathrm{MPa})$ 